\title{
DIE INSCHRIFTEN DES ERSTEN TORES VON PTAH TEMPEL IN KARNAK \\ von
}

MAHMUD ABDEL RAZIQ - Sherin Hafiz

\section{TOR}

Mahmoud.awad@tourism.suez.edu.eg

Die Turme des ersten Tores sind je X m breit und Xm lang. Sie haben Nischen in den einander gegenüberliegenden Seiten. Die erhaltene Hohe des linken, nordlichen Turms beträgt xm, die des rechten, sǜlichen Turmes xm. Baudatum: Ptolemaus Vl.

Nordturm, Westseite:

Vier Register, in denen jeweils der König opfernd vor Götterpaaren abgebildet steht. Er trägt den kurzen, spitzen Rock und verschiedene Kronen; hinter ihm jeweils die Schutzformal: s3 c\#\#n x\#\# w3s nb ji\#\#3. f mj Rc\#\# u\#\#t “ Aller Schutz, Leben und Glück sind hinter ihm wie Re, ewiglich "Den unteren Abschluss bildet ein Fries als Lotos- und Papyruspflanzen. Die Bilder sind in versenktem Reliefgearbeitet.

Oberstes Register: Die Titulaturen des Königs und der Götter sind zerstört. Teile der Ritualbeischrift sind erhalten:

Text vor dem König:

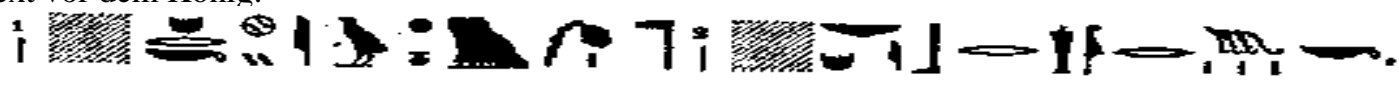

“(.... wie ..... ist doch $)$ der Duft, mehr als der Geruch eines Gottes. (Empfagne) den Duft echten jbr- Oles auf deinem Haar."

Text vor dem Gott:

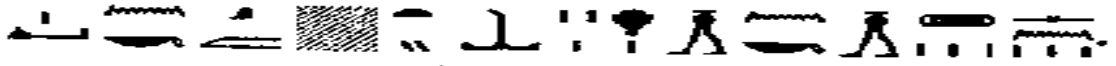

[" Ich trage Sorge für dich, dass die Bewohner des weihrauch-

lands dir ihre Abgaben liefern"

2. Register:Der König, mit xy- Krone, langem Kopftuch und kurzem spitzem Rock opfert eine Schreiberplatte vor Ptah und Maat.

Horizontale Textzeile über der Szene:

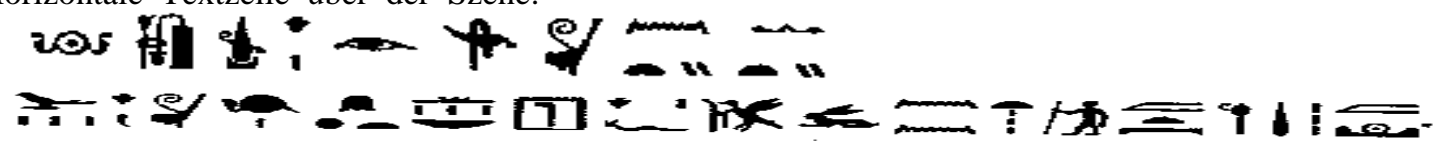

"1 Wohlgemuter Schreiber, Leiter des Seienden wie auch des nicht Seienden, Herz des Re, das alle Dinge erdachte- den gottlich ist sein Herz das das Seiende erschuf- Fürst im Himmel. Befehlshaber im Horizont."

Titulatur des Königs:

"Theoi Philometores, Sohn des Maat, Beschutzer dieses Landes"

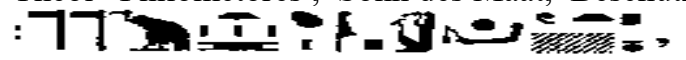

Titulatur des Gottes:

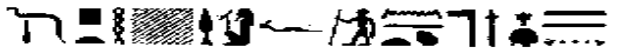

“'Ptah, der unter seinem Ölbaum, Fürst der Maat, Großer Gott,. Öberster der beiden Länder ”

Titulatur der Göttin:

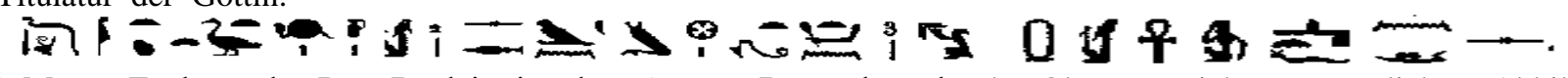

“Maat, Töchter des Re, Begleiterin des Amun, Brustschmuck des Obersten Richters, Herrliches Abbild dessen, der seinen Namen verbringt, der lebt und zufrieden ist, wenn er sie erblickt."

Text vor dem König:

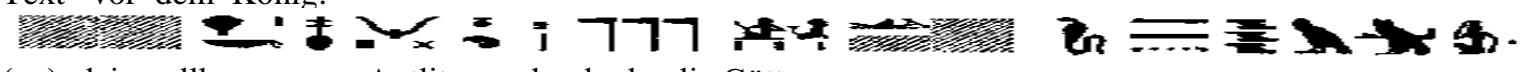

(...) dein vollkommenes Antlitz, wodurch du die Götter

und Menschen getrennt und die beiden Lānder und ( andere) Lānder

voneinander unterschieden hast."

Text vor dem Gott:

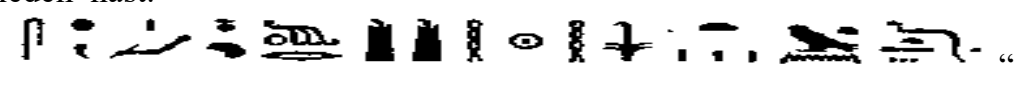

Ich zeichne Dir eine Lebenszeit bis in alle Ewigkeit auf und eine bedeutende Königsherrscherschaft für die Unedlichkeit."

Text vor der Göttin:

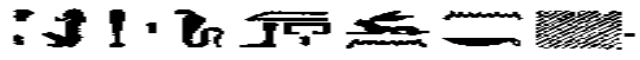

“ Ich schliesse mich Deiner Majestāt an, wo du auch sein magst, Ohne mich einen Augenblick von dir zu entfernen.

3. Register:

Der König, mit Atefkrone, opfert ein Pektoral vor Month und Ra' attui.

Horizontale Zeile oben:

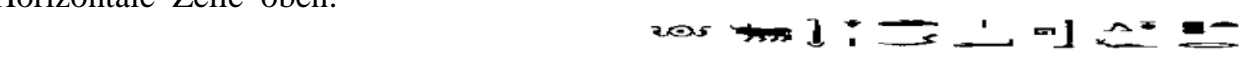

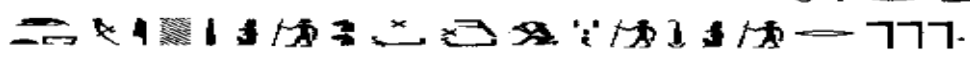


“ Stier der mit kühlem Mut, der mit (den) zupakkendem Arm, wenn er auf das schlachtfeld getreten ist, Merti, der mit großer Kraft, der seine Feinde schlägt, Werti, der großer als die Götter ist."

Titulatur des Königs:

\section{$77 \div \div 3$}

" Theoi Philometores, Vollkommener Gott, Horus, der mit großer Kraft."

TitulaturdesGottes:

\section{à}

Month- Re, Herr von Theben, Amun- Re in (...) Theben als Amun, der Herr Alteste, Herr von Edfu in (...)" Titulatur der Göttin:

\section{is 을}

"Ra,attaui, Erste von Theben als Neith, Mütter des Aton, die den

Selbsterkenner im Grosse gebiert."

Text vor den König:

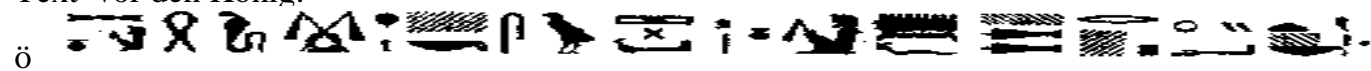

Ich habe dein Schutzamulett geknupft, sodaß es mit deinem Pectoral ist. Ich habe die beiden Armbänder aufgereiht, um deine Glieder zu schützen."

Text vor dem Gott:

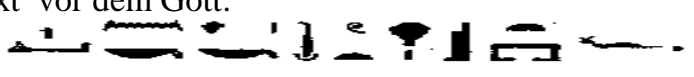

"Ich trage Sorge für Dich, dass Dein Herz an seiner Stelle bleibt."

Text vor der Göttin:

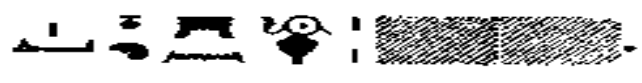

"Ich trage Sorge für dich, dass das Pektoral des Re

Unterstes Register:

Der König, mit unterāgyptischer Krone, opfert ein Feld vor Amun

und Mut.

Horizontale Zeile oben:

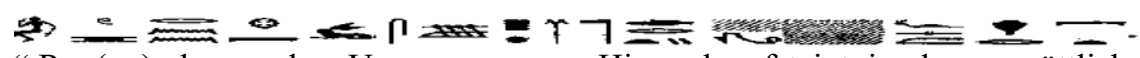

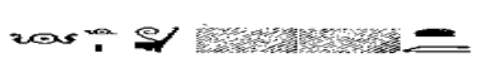

"Re (...) der aus dem Urgewasser zum Himmel aufsteigt, in dessen göttlichen Augen das Leicht existiert, wenn er sich zum Himmel begibt."

Titulatur des Königs:

" König von Ober- und

Unterägypten NN, Sohn des Re NN

Südturm, Westseite

Darstellungen wie auf dem Turm links; allerdings sind nur

2 Register erhalten; vom dritten Register, oben, existiert nur noch ein geringer Rest.

Oberes Register:

Der König, mit kombinierter U.A. und Atef- Krone opfert vor Chons und Mut.

Horizontale Inschrift oben:

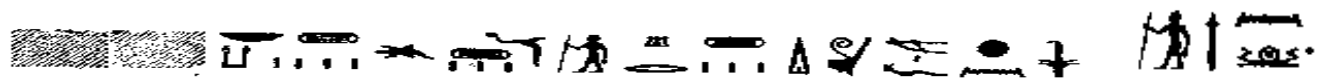

“(... ) Liebe, Herr der Speisen, der reich an Speisen und enorm an unterhalt ist, der dem gibt, den er liebt, Chons, Gewaltiger der Sonnenbarke."

Titulatur des Königs

“(...) NN, ( Sohn Re ) NN (Ptol. Vl)"

Titulatur des Gottes"

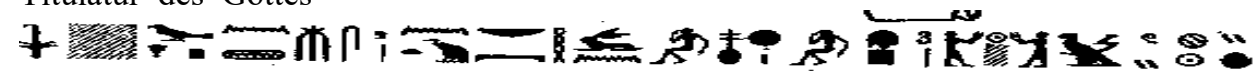

"Chons, das grosste, alteste und erste Kind des Amun, Kind der Mut, der Herrin des Himmels, vollkommer jungling, der im Morgengrauen eines jeden Tages immer wieder auf neue jung ist"

TitulaturderGöttin:

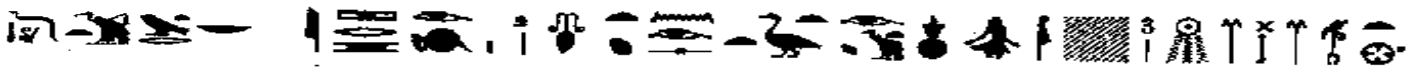

“Mut, Grosse, Herrin von Ascheru, Auge des Re, Mütter dessen, der sie zeugte, und Töchter dessen, der den Sohn der Mut zeugte, die, die das Licht wiederholt in Theben gebiert."

Text vor dem König: 


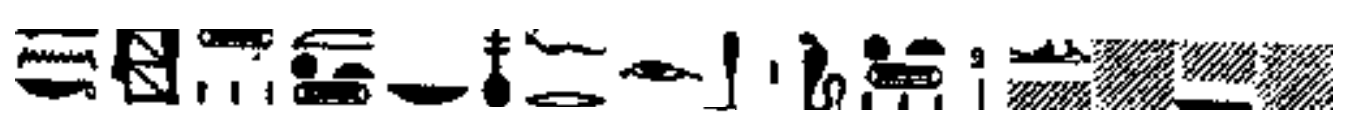

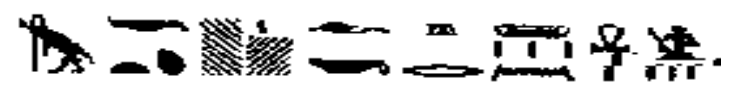

“Empfange das aus allen guten Dingen bestehende Opfer, auf dass deine Majestät davon Mahlzeiten mache (...), auf dass du die Göttinnen ( mit Speisen) versorgst und den Unterhalt für die Lebenden bereitest."

Text vor dem Gott:

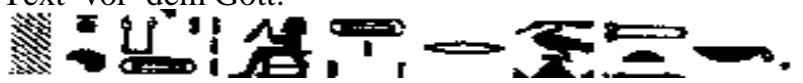

"( Ich trage Sorge) für dich, dass Nahrung und Kostlichkeiten deine Tafel überschwimmen."

Text vor der Göttin:

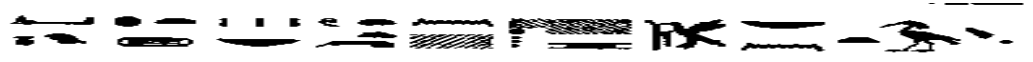

" Ich überlasse dir alle Dinge, die der Nil erzeugt und alles, was das Freuchtland hervorbringt."

Unteres Register:

Der König, mit oberägyptischer Krone, opfert ein Feld vor Amun und Chons.

Horizontale Zeile oben:“

$$
\text { 2. }
$$

üDer, der den Himmel auf den vier Stützen erhob, der die Erde auf ihren beiden Fundamenten festsetzte, der die Gebirge erschuf und das Meer enstehen liess, der, für den alle Dinge auftauchten, der, der ohne einen Anderen bei sich, alleine kam, Amun, Grosser, Schopfer aller Dinge.

Titulatur des Königs:

$$
773 . T_{1}
$$

"Theoi Philiometor

es, (...) Himmel und Erde (...) der selbst entstanden ist.”

Titulatur des Amun-Re

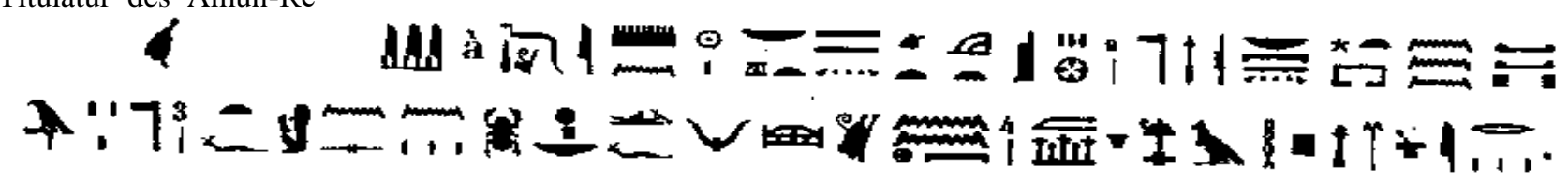

Amun- Re, Herr der Throne der beiden Länder, Erster im Tempel von Karnak, Grosser Gott, Herr uber Himmel. Erde, Unterwelt, Gewasser und Berge, Gott der Götter, Vater der Synnaoi, aus dem alles, was entstanden ist, entstand, der, der den Schrein des Himmels zu Beginn offnete und danach die Topferscheibe konstruierte."

Titulatur des Chons:

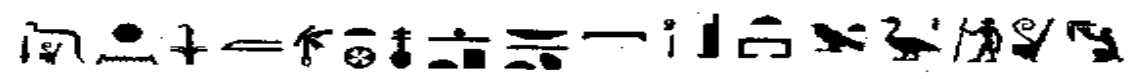

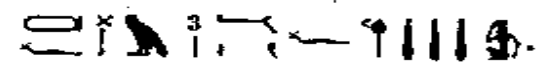

Chons aus Theben, Nefer Hotep ,Herr der Maat. Uberster des Allerheiligten, ältester, der seinen Namen verbringt, der, dessen Zunge seine Befehle wieder gibt.

Text vor dem König " Ich erweitere (...)."

NORDTURM, INNEN, WESTLICH DER NISCHE

Vier Register, ein Register mit Nilgötterprozetion. Der König, mit verschiedenen Rocken und Kronen, opfert je einen Gott. Hinter dem König jeweils die Schützformel.

Oberstes Register:Der König, mit langem Rock vor zwei Göttern. Die Szene ist beinahe ganz zestört.

Text vor der Göttin ( rechts):

"(...) die Lebendzeit des Re im Himmel"

2.Register:

Der König in kurzem Rock und Osiriskrone auf der Bautelperücke erhebt zwei Stoffstreifen vor Nefertem. Horizontale Textzeile oben:

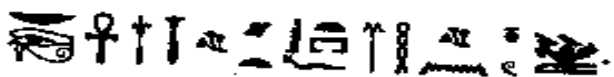

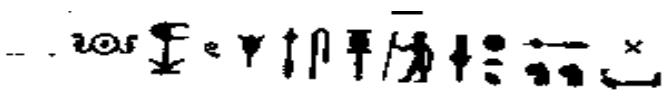

“ Grosser Lotus, Freund der Sachmet, Kraftvoller, Herr des Auges der Leben der beiden Länder” ( Hathor), Beschutzer, dessen Platz an der Spitze der Sonnenbarke ist."

Titulatur des Königs: 
$\exists 7 \stackrel{=}{\Rightarrow}$

"Seinem Vater die heilige Binde geben" ( Name des Kgs fehlt?)

Titulatur des Gottes:

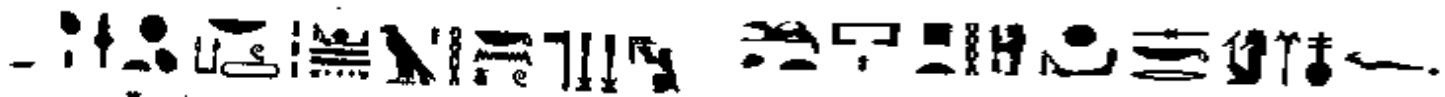

“ Nefertum, Sohn der Sachmet, Herr der Speisen, Beschützer der beiden Lǟnder, Horus-Hekenu, Gott der beiden Genossen, der mit verborgenem Geheimnis, Erster des Ptah-Tempels, Beschützer des Sokar in vollkommenheit."

Text vor dem König:
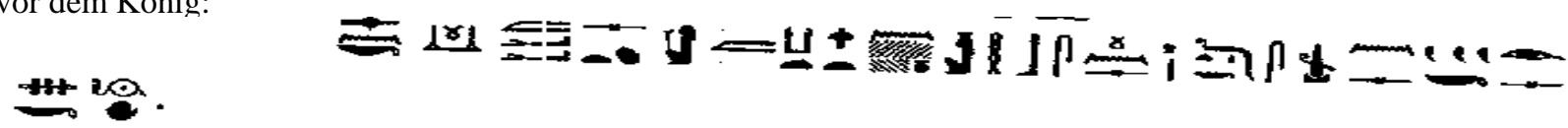

"Empfange die Leinwand aus den Händen der Einzigen als verzugliches Werk der $(\ldots)$, auf dass sie deinen Leibbekleide, deine Glieder umgebe und jeden Tag deinen Schutz bilde."

Text vor dem Gott:

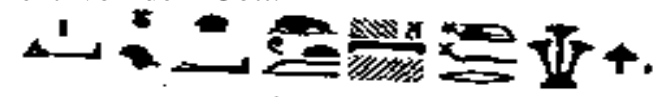

"Ich gebe dir die Krone, die als (Verbindung (?) von ) Himmel ( und Erde ) erscheinet, wenn sie vom Hinterkopf hervortritt.

3.Register

“ Der König, mit O.A. Krone auf der Lockenperucke und kurzem, spitzem Rock, opfert die beiden Länder vor Min-Amun.

Horizontale Textzeile oben:

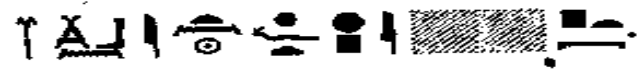

" Abbild des Re, Prachtiges Bild des Harsiese, den man an der Stelle des Aton Kronte, gemäss dem werden der ( beiden Hälften ) des -Himmels."

Tilulatur des Königs:

"Seinem Vater Ober- u d Unterägypten Geben." (fehlt den Name?)

Titulatur des Gottes:

"Amun von Luxor (...) dort als Amun in der (verborgenen) Kammer."

Text vor dem König:

$$
\equiv-7-\overline{1}= \pm
$$

$=\rightarrow 1 \leq 1 \leq 10$

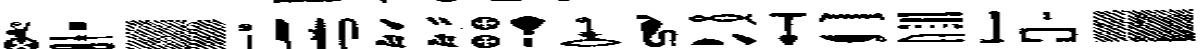

Empfange den Papyrus, ich reiche dir Binse, Ober- und Unterägypten, die (beiden) Teile (...), die beiden Palastteile ( ̈̈gypten) südlich und nodlich von dir. Du hast beiden Länder Vereint, wo (... )."

Unterster Register

Der König, mit xy- Krone und kurzem spitzem Rock, opfert Brot vor Ptah.

Horizontale Textzeile oben:

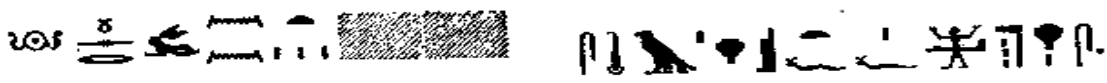

Verkunder des Seienden, (...), Horus ist an die Stelle seines Vaters gesetzt, sodass die Götter sich darüber freuen."

Titulatur des Königs:

“ (..) ( König von Ober- und Unterägypten), Herr der beiden Länder NN, Sohn des Re, Herr der Kronen NN ( Ptol. X111).

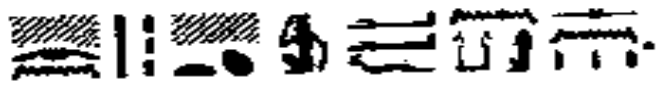

Hinwenden (spr) zu den Göttern und Göttinnen, Erheben Seiner Arme zu ihrem Ka."

Titulatur des Gottes:

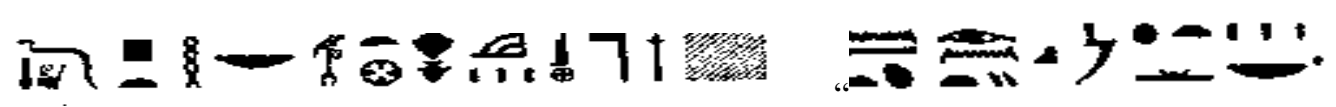

"Ptah, Herr von Theben, der im Tempel von Karnak befindlich ist, Grosser Gott, (Besitzer) der Maat, der macht was ist, und jede Sache erschafft."

Text vor dem König: 


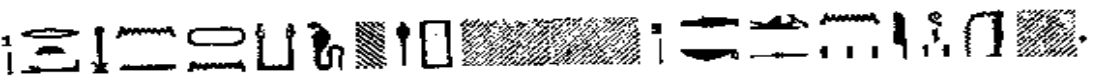

“( Ich) uberreiche deinem Ka Opferbrot, Weinbrot (...), auf dass du davon (empfangest), indem sie makellos sind."q

Text vor dem Gott:

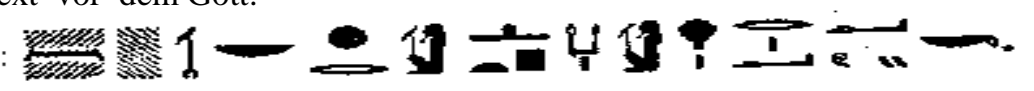

"Ich gewahre dir alles Leben, alle Dauer und alles Wohlergehen durch mich, auf dass mein Ka mit dem (,was) aus deinen Handen ( kommen sei)."

Subregister:

Nilgotterprozession. FEHLT

Südturm, innen

Die Registereinteilung entspricht der gegenüberliegenden Seite.

Vom obersten erhaltenen Register sind nur noch geringe Reste Sichtbar.

2,Register:

Der König mit xy- krone und xy- Perucke opfert vor Horus.

Horizotale Textzeile oben:

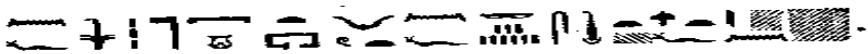

$\cos 1=\mathrm{KKK}-1 \div= \pm$

“ Herrscher der beiden Landestelle, mit dessen Namen, die beiden Länder beschriftet sind, König der Götter, Oberster des Thrones, für den die Neunbogenvolker vereinigt sind, dessen Schatten (..) ruht."

Titulatur des Königs:

?? "Die weisse Krone der Nechbet und die Rote Krone der Uto"

Titulatur des Gottes:

$$
\text { 9а ג' }
$$

"Horus, Sohn der Isis, Sohn des Osiris, Herrscher Erbe des Wenennefer (... )"

Text vor dem Gott:

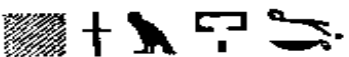

“ (....) dein vermachtnis.

Nordturm, innen, östlich der Nische;

Ich bringe dir dar (stwt=) $n=t$ ) das Anch-Gefass als Besitz (xwd) Des Herrn (..)

(..) als Herkenu aus reinem Gold als Opfergabe aus den Handen ihrer Mütter. Ich bringe dir dar kostbaren, geschliffenen Stein (hhj dtj) und neuen, harten Stein (bnw $n \mathrm{~m} 3 \mathrm{w}, \mathrm{t})$ (...) 2.) (aus) (...). Ich bringe dir harten Stein (bnw), damit er seine gekeime Geste (bs3) zu Pulver zermahle. Ihr Sohn befindet sich im Innern, einzige Strahlende, um den Himmel als Sonnenglanzen der $(<\mathrm{ndw})$ zu befahren.Ich bringe dir dar die gekeime Gerste (bs3) dieses Opfers als qsn.tj - Herz aus der Hand des jenegen, der die beiden Länder leitet.Ich bringe dir dar das Silphium dieses Opfers als reines Ssk3 aus Asien (st.t), damit du vereinigst (...)

Ich bringe dir dar das Wasser dieses Opfers als Wasser (smswn) von xntj-3x.t aus den Händen der jr-m-mr. ${ }^{2}$ Ich bringe dir dar die Maische (šbb.t) dieses Opfers als Weihrauch und Merrhet, 3.) (...)(Ich bringe dir dar) (...) als xm in einem Napf aus dem Salbenlaboratorium. ${ }^{3}$ Ich bringe dir dar seine Datteln und seine Dattelmasse als Maische ( šbb.t) die aus gerostetem Getreide (<gw.t) gehartet ist, aus den Händen der Troglodyten (Jwn.t jw).Ich bringe dir dar den Hönig dieses Opfers als Weihrauch (shtp) aus xntj- 3x.t), das überquellt $(\mathrm{msms}=$ gsgs $)(\ldots)$ fünf Gewasser der beiden Herren.

Ich bringe dir dar das srf- Wasser dieses Opfers als Wein des Herrn von Buto (Jm.t) aus den Händen des Fnxw Volks.Ich bringe dir dar wohlreichende Substanzen (x3) ( als Myrrhe) ${ }^{4}$ undWeirauch (htp) zu seinem Schutz (...)Ich brige dir dar (...) seinen Emmer (bd.t) für die Mütter des Herrn der Götter, die Flammende $^{5}$ (nsr.t) der $\mathrm{Ba}<\mathrm{s}$ von Heliopolis. Ich bringe dir dieses Opfer selbst dar als Grosser, an der Spitze der Neunheit, gewaltig anStimme im Kollegium des Geb.

4.) (...) hervorgebracht aus Lapislazuli und die Salbe $(<m<. t)$ dieses Opfers besteht aus trefflicher (iqr) Myrrhe (<ntjw) der Majestät, die an den Scheitel (sm3) der Sänger und Sängerinnen gegeben wird. Es kommt der König, Herr der beiden Länder NN, und er tanzt.

Er kommt, und er Lobpreis.

- $\quad$ seine Fürstin, sieh wie er musiziert;

- O Gemahlin des Horus, sieh wie er tanzt!

- Der Sohn des Re Ptolemaios Xl. mit gewaschenen Händen und mit reinen Fingern. ( O seine Fürstin ) sieh wie er musiziert;

O Gemalin des Horus, sieh wie er tanzt! ( Er) bringt dir dieses Opfer dar. ( O seine Fürstin, sieh) wie er musiziert; O Gemalin des aufrecht, wie er tanzt! Sein Herz ist aufrecht, er ist aufrichtig ${ }^{6}$ und keine Dunkelheit ist in Seinem Herzen. 
5.)(O Goldene, wie schön ist dieses Loblied; es ist wie das Loblied des $)^{7}$ Horus selbst! Es ist der vollkommene Gott,Der Herr der beiden Länder NN, beim Lobpreisen. Ihm zugehörig sind deine Gefolgsleute.Es ist dein kleines Kind, dein Ihi, Nicht lasst er jemanden durch sein Wort schifflos sein.Nicht vermindert er dein Opferbrot. Sein Herz ist aufrecht, er ist aufrichtig, und keine Dunkelheit ist seinem Herzen.

Sein Abscheu ist die Trauer, die deinen ka befall; Sein Abschau ist Hungern und Dursten; (sein) Abschau ist (...) der Sonnengöttin. Nicht wird sein Brot geweirauchert (pd) auf seinen Händen.( die Speisen), die hervorkommen aus dem Auge des Horus. Er reinigt sie und bringt sie dir dar. Er kommt, um zu musizieren, und er kommt, um zu tanzen. Sein Gefäss aus Blumen, sein Korb aus Binsen, sein Sistrum aus Elektron und sein Menit aus südlichem Grundstein.Er lässt seine Beine springen fûr die Herrin der Musik, er tanzt für sie, sodass sie seinen Zustand liebt.Es kommt Ihi, das Kind, ( Ihi ) - Priester der Hathor, seiner FurstinSeiner Fürsten an diesen Tage, an dem sie sein Unheil verhullt und seine Schuld lost

8. (..) die Götter. Er wendet die Herz der Menschen (rxj.t) ( zum Guten). Es kommt der (Ihi-) Priester der Hathor, seiner Fürstin. Ihr Vorrathaus besteht aus Speisen, aus Vogel und Fischen(...) aus allen guten Dingen.

- O wie schön ist es, wenn sie kommt! O wie schön ist es,

- wenn sie ruht!

- O wie schön ist dein Antlitz, wenn es zufrieden ist! O wie schön ist dein Antilitz, wenn es zufrieden ist ! Dein Finger sind gelöst, wie schön ist die, die ruhrt, Hathor, Herrin der Götter !

Deine Finger sind geofnet, und offen ist der Hals; Alles Enge ist gelöst, gelöst zu seiner Zelt.

$\mathrm{Du}$ offnest die verstopte Nase, und läst die Fesseln (g3s) (...)Du erlost auch den König, den Herrn der beiden Lännder NN, Sohn des Re, Herr der Kronen Ptolemaios Xl. Du erlost ihn von allem schlimmen Unheil, den sie handelt dagegen ${ }^{8}$ durch dieses und alles mögliche andere, ${ }^{9}$ Das, was du ihm gewährt hast (htp), möge auf dich zurükfallen ( $r=t) O$ seine Fürstin,, als diese deine schönen Opfergaben.

Du gibst dejenigen, den (du) liebst Dieses ist täglich fest eingerichtet (als Ritual) im Tempel der Hathor, in Theben befindlich, der Isis, der Grossen, Der Gottesmutter, des Auges des Re, Der Herrin des Himmels und Fürstin der Götter,

Text vor der Göttin:

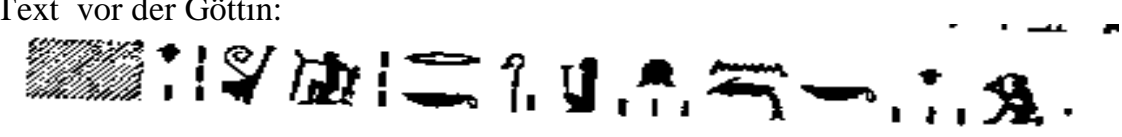

“(...) die Herzen) der Feinde von dir, ich beherrsche die Gedanken ( m3tw) der Empörer.” Mehrere Register. Der König opfert vor verschiedenen Göttern.

Oberstes Regiter:

Der König, mit xy-Ornat, opfert xy vor xy

Text vor dem König:

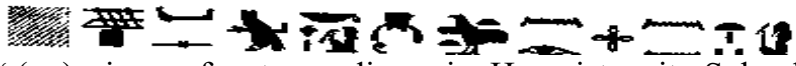

"(...) sie empfangt von dir, mein Herz ist weit; Sohn dessen, der alles Seiende schuf."

Text vor dem Gött:

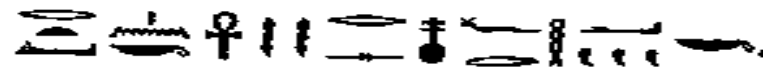

“ Ich gewăhre dir ein angenehmes Leben, um deine Glieder zu vervollkommen.”

2.Register:

Der König, mit xy-Ornat, opfert xy Re Harachte (?)

Horizontale Textzeile oben:

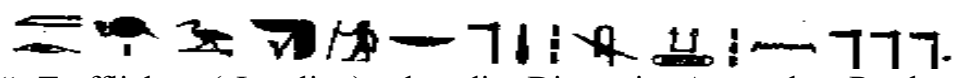

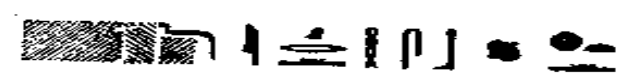

“ Trefflicher (Jungling), der die Dinge im Auge des Re berechnet, Grosser Thot, Herr der Göttesworte, Darbringer der Speisen für die Götter.”

Titulatur des Königs:

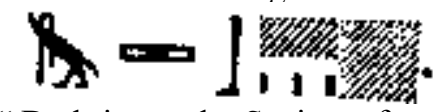

"Darbringer der Speiesen für (...)"

$\mathrm{Ti}$

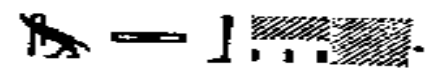

Titulatur des Gottes:

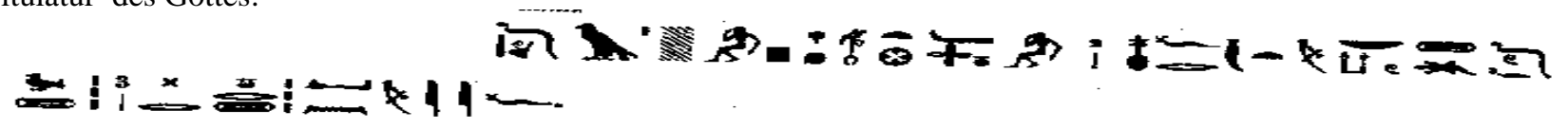

“Horus-Re, das Kind, zu Gast in Theben, Herrlicher Jungling, Heissgeliebter, Herr der Speisen, der reich an Nahrung und

Bedeutend an Bedarf ist, der dem gibt, den er liebt." 
Unterstes Register:

Horizontale Textzeile oben:

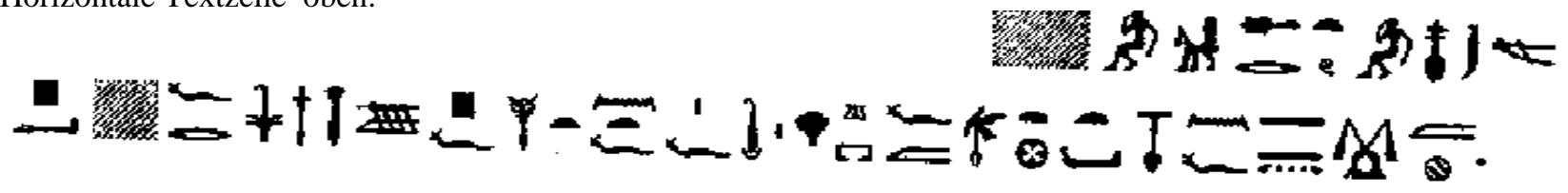

"Herrlicher (knabel), vollkommenes Kind, Heissgliebter, von

seinem/r (..) zum König der beiden Länder Geborener. Er möge die wurde seines Vaters annehmen und auf seinem Thron Sitzen, nachdem er die beiden Länder zu einem geeint hat."

Titulatur des Gottes:

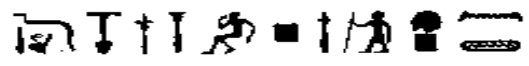

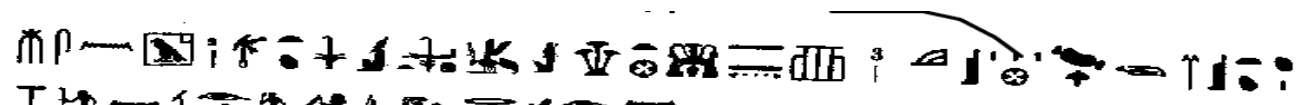

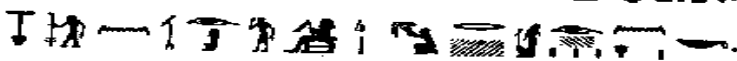 \\ "Somtus, das Kind, Gewaltiger, Erster des Amun, geboren von Hathor von Theben, König von Unterägypten, vereiniger der beiden Länder, Erster des Tempels von Karnak, Trefflicher Erbe, Spross aus der Isis, altester Sohn des Osiris, begnadetes Kind des jenigen, der seinen Namen verbringt, Granat der versorgung für jedermann."}

Text vor dem König:

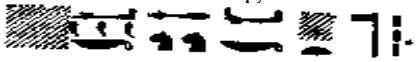

(...) deine Glieder. Gross ist deine Kraft, Erster der Götter.”

Text vor dem Gott:

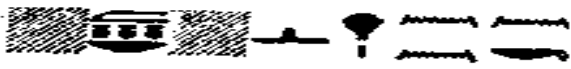

(...) in jeglichen Herzen. (Die beiden ) Länder ) sind mit ( ihrer) Abhangigkeit von dir zufrieden."

Subregister : In der rechten, östlichen Seite der Nische ist in xy-Zeilen ein Hymnus auf den Gott Ptah angebracht.

Text:

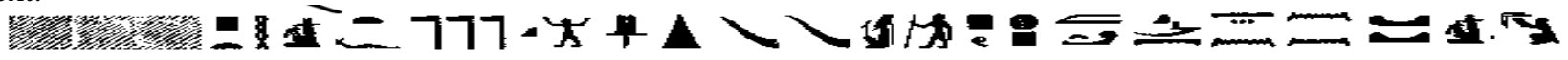

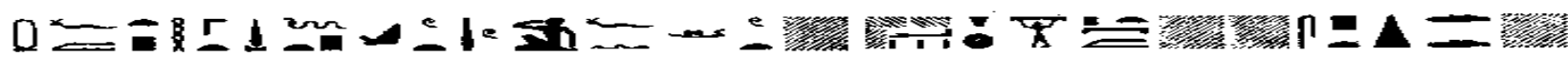

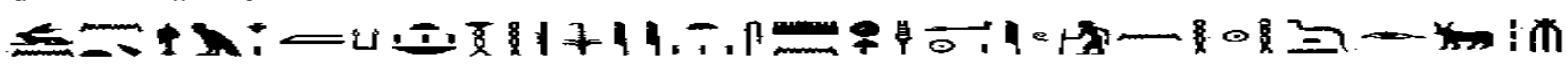

$$
\begin{aligned}
& \text { P.三 - }
\end{aligned}
$$

“( ... ) Ptah- Tatenen. Götter vater, Der mit hohem Federpaar und spitzen Hornern. Er ist der Alteste, der zu Anbeginn enstand Schöpfer von Himmel und Erde, Gewässer und Bergen,Der seinen Namen in der Höhle der Unterwelt verbirgt, Dessen Worte man hört, ohne (...) zu erblicken. (...) die grossen (...) Der den Himmel erhebt als Werk (....), Der beim Schaffen des Seienden Geschickte, Der bei juglichem Werk Weise Der das Königtum dauerhaft Begrundende, Der die Zeit Vervollkommende, Der Greis von Ewigkeit und Unendlichkeit, Der Stiere Zeugende und Kuhe Gebarende Der die Samen der Götter Erschaffende. Herr der Maat, Das zu kommen Prophetzeite (....). (.....) Herr der Wurdeverleihung an die Ka,s Der Wurde verleihende (...)---

Ausser mit dem Ptah- Hymnus ist die Nische mit horizontalen Textsreifen Dekoriert. Sie enhalten abwechseind: Titulaturen Ptolemaus V1.

2.) Auf nb- Korben stehene ankh-Zeichen, die mit Armen rechts und links je w3s-Szepter halten

3.) auf nb- Korben sitzende Kartuschen zwischen Schlangengottinnen und Millionenjahrgötter mit Jahresrispan, alternierend.

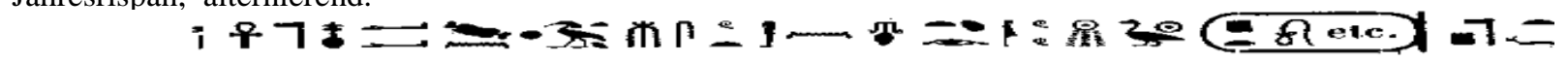
$\doteq 13 x-1=1$

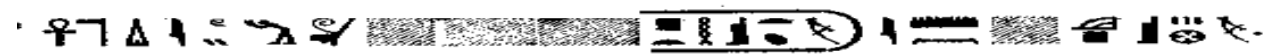

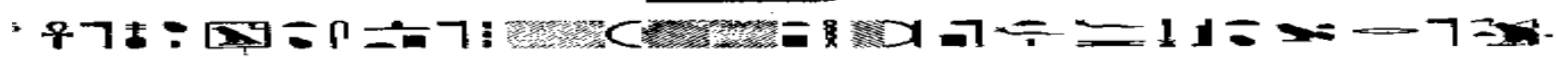

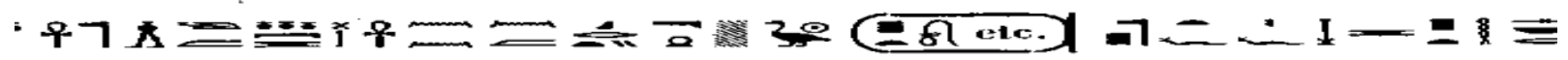

$\rightarrow 10$

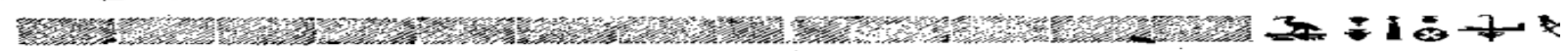

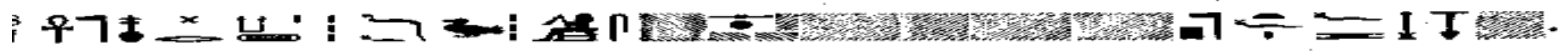

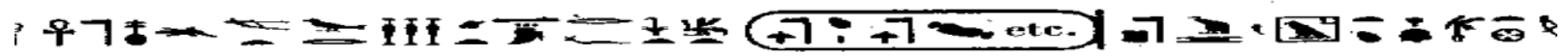

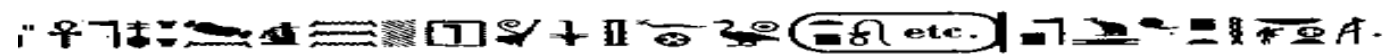

Texte der Titulaturen: 
Oberste erhaltene Zeile: “ Es lebe der vollkommene Gott, Erbe der Strahlenden, Abbild der Mutter, Lichtschopfer, Sohn des Re Ptolemeios Theos Philopator Philadelphos, Geliebter der Mut, der Grosser, der Herrin von Ascheru."

1. Zeil: Zerestört

2. Zeile: "Es lebe der (vollkommene) Gott, der (seine) Mutter lobpreist, (der Sohn des Re Ptolemaios) Geliebter von Ptah und Isis, Geliebter des Amun, des (Ersten) im Tempel von Karnak."

3. Zeile: "Es lebe der vollkommene Gott, Sohn der Hathor, der die Götter zufriedenstellt und den Göttinnen ans Herz gewachsen ist, ( Sohn des Re

Philopator Philadelphos, Geliebter der Isis, Grossen, der Gottesmutter.”

4. Zeile: "Es lebe der vollkommene Gott, Bringer dessen, was aus dem Nun er- wachst, Wiederbeleber der Nilflut aus den beiden Quellochern, Sohn des Re Ptolemaios Theos Philopatos Philadelphos, Geliebter des Ptah-Soker-Osiris."

5. "Es lebe der vollkommene Gott, Gerechtigkeit Ausübender, (...) der die Götter mit dem, was sie mögen, zufriedenstellt, (...). Geliebter des Thot, zu Gast in Hermonthis.”

6. Zeile: -" Es lebe vollkommene Gott, Nahrungs- und Speisenreicher, Ausstatter der Tempel mit Speisen, (...) Theos Philopator Philadelphos , Geliebter des Somtus.”

7. Zeile: " Es lebe der vollkommene Gott, Vielgeliebter, Vorderster der ganzen Erde, König von Oberund Unterägypten NN Theos Philometor, Geliebter derHathor, der Obersten von Theben."

8. Zeile:“ Es lebe der vollkommene Gott, Erbe des Tatenen, heiliger Same des jenigen, der sich südlich seiner Mauer befindet, Sohn des Re Ptolemaios Philometor, Geliebter des Ptah, des Herrn von Theben."

Text:

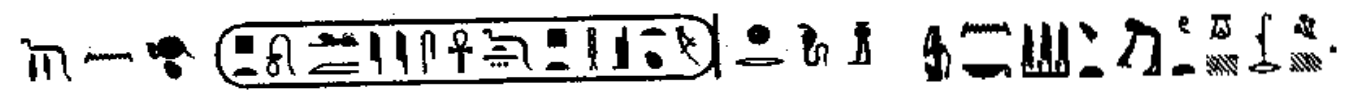

"Rede : Es kommt zu dir der Sohn des Re Ptolemaios, Geliebter des Ptah und der Isis, er bringt dir das Feld, gebeugt unter Pflanzenwuchs."

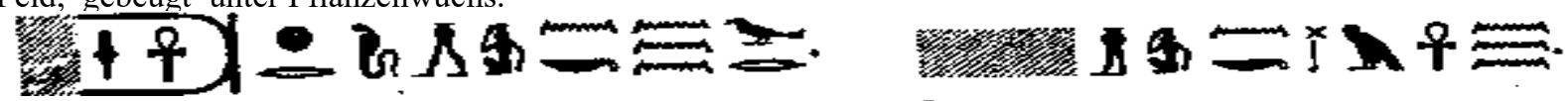

“(Rede: Es kommt zu dir ( der König von Ober- und Unterăgypten, trefflicher- Erbe- des- Gottes-und - der Gottin, der - seine- Mutter- die Beschuzerin- liebt, Erwahlter- des- Ptah, Garant-der-Ordnung-Ordnung- des Re) Leben-des- Abbild-des-Amun) ( WO GEHT DIESE ECKIGE KLAMMER AUF?) (WAS SOLLEN DIE BINDESTRICHE?), er bringt dir die grosse Flut (...).” “( Rede : Es kommt zu dir ...), er bringt dir die wiederbelebung der Nilflut."Die Reliefs und Inschriften dieser Wand sind beinahe vollkommen zerestört.

NORDTURM, OSTSEITE

Die ins Tempelinnere weisende Turwange trägt vier Register, die den König opfernd vor jeweils zwei Göttern zeigt. Er trägt den kurzen, spitzen Rocke und verschiedene Kopfbedeckungen.

Oberstes Register:

Der König, mit xy- Krone auf dem langen Kopftuch, opfert Weihrauch vor Min-Amun und Ptah.

Titulatur des Königs:

“König von Ober- und Unterāggypten, Herr der beiden Länder NN, Sohn des Re, Herr der Kronen NN." ( Ptol.Vll.)

Text vor dem König:

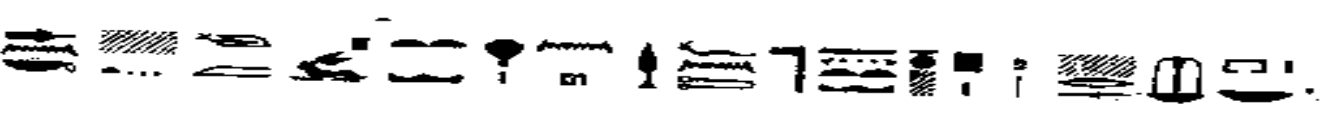

“ Empfange die aus Punt von ihrem Baum aus dem Gottesland stammende Myrrhe, (...) um deinen Tempel festlich zu machen.'

Text vor dem Gott:

."Ich uberreiche dir die Wüste und alles was in ihr ist."

Text vor der Göttin:

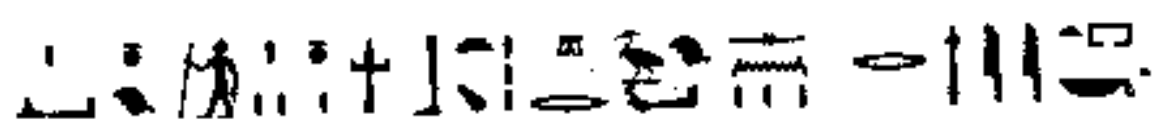

“ $\mathrm{Zu}$ deinen Gunsten uberantworte ich die Grossen der gesammten Erde und ihre Stuerertrage deinem Tempel."

1. Register:

Der König, mit kombinierter U.A. und Atefkrone übergiesst einen Opferstand mit Weihwasser; vor ihnen Osiris und Isis.

Horizontale Textzeile oben;

vor

“ Der König als sein Leib, Schöpfer der Weissen Krone beidem die beiden Kroneschlangen sind, Herr davon in den gesamten Gauen, die in ihnen Befindlichen gehören zu ihm als seine Untertanen ( rxjt) und seine Schwester und Gemahlin ist ihrer aller Herrscherin.” 
Titulatur des Königs:

"' König von Ober- und Unterāgypten NN, Sohn des Re NN, Theoi Philomatores, sein geliebter Sohn, der für seinen Vater nûtzbringen ist."??

Titulatur des Gottes:

国 I7

“Osiris- Wenennefer, könig der Götter, Herrscher der Neunheit, Grosserim Gau von Abydos Einziger Herr in Ägypten.”

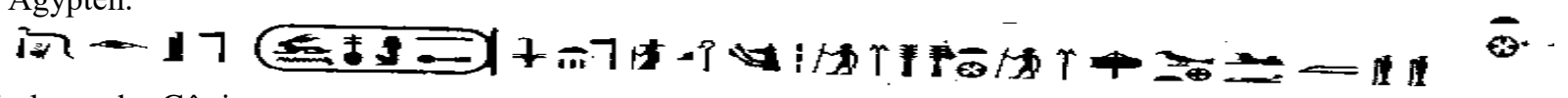

Titulatur der Gôttin:

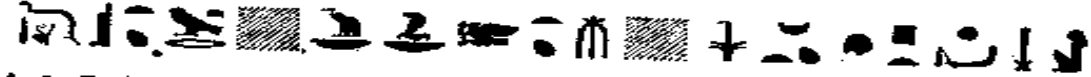

“ $\overline{\text { Isis, }}$, Grosse , Gottesmutter, Kônigin des Volkes, Mutter des Herrschers, Oberste Königsgemahlin, Beschutzerin ihres Bruders."

Text vor dem König:

\section{$-112$}

“ Seinem Vater Wasserspenden darbringen."

Text vor dem Gott:

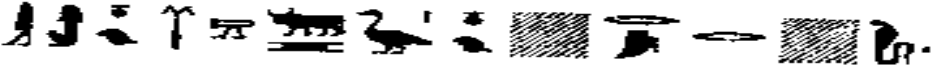

"Ich komme zu dir und fuhre debei den Schwarzen Stier vor. Für dich Uberflutet (...) soviel du willst."

Text vor der Gôttin:
$\mathrm{B}^{2}$
$\mathbf{S}=\ldots$
$=\underline{-2}$

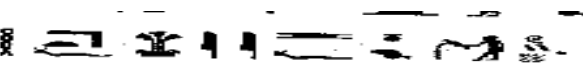

"Ich Befruchte für dich den grossen Nun in seiner Hohle, auf dass er für dich (alles), was überflutet werden muss, uberschwemme."

2. Register:

Der Kônig, mit blauer Krone, darauf die Sonnenscheibe erhebt einen Spiegel vor Hathor und Horus.

Horizontale Textzeile oben:

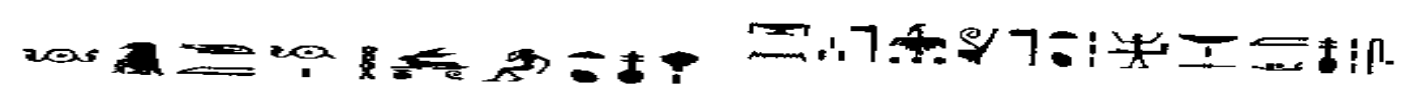

"Prachtige, die aus Re hervorgegangen ist, Mädchen mit vollkommenem Antilitz, deren Herr sich Freut, wenn er ihre Schônheit sieht."

Titulatul des Kônigs:

“ Theoi Philometores, Zweiter des Tatenen, Dritter des Kônigs ka.”

Titulatur der Gottin:

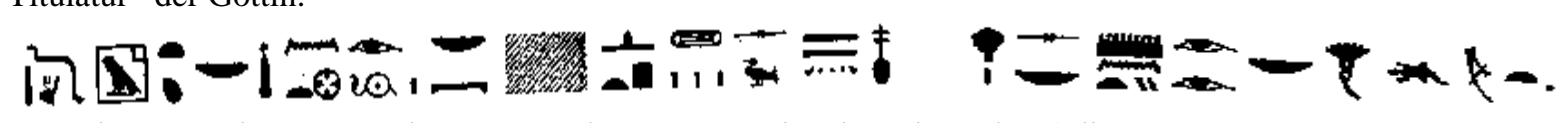

" Hathor, Herrin von Dendera, Auge des Re, Herrin des Himmels, ( die reich) an Opfern ist, die beiden Länder speist, die mit schönem Geschicht, die mit festlich geschminkter Augenpartie, Herrin der Susse,Vielgeliebte."

Titiulatur des Gottes:

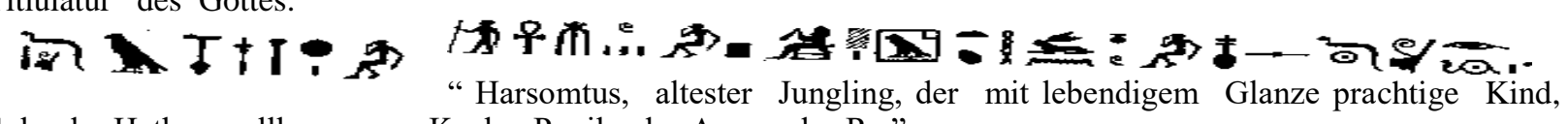

Sohn der Hathor, vollkommener Knabe, Pupile des Auges des Re."

Text vor dem König:

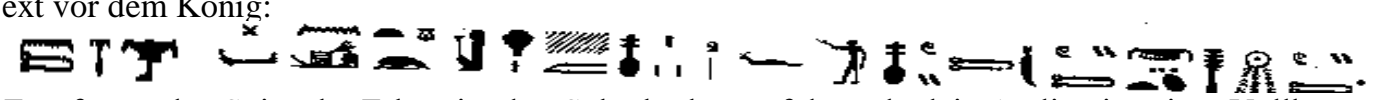

"Empfange den Spiegel, Erbaurin der Sokarbarke, auf dass du dein Antlitz in seiner Vollkommenheit erblikest. Oh, wie schön bist du, wie suss bist du, Goldene, wie glanzend bist du !"

Text vor der Gôttin:

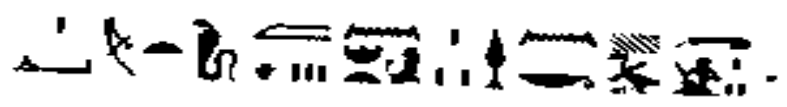

"Ich trage Sorge dafûr, dass Liebe zu dir in den Herzen der Frauen sei, und das den Mannern dir gegenûber Frauendlichkeit zu eigen sei."

Text dem Gott:

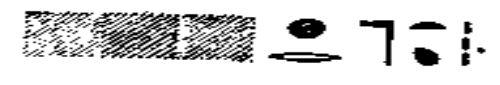

“(...) bei den Gôttinnen." 
Unterstes Register

Der König mit blauer Krone, erhebt die Arme vor Amun und Mut. ûber ihm die Sonnenscheibe mit zwei Uraen.

Horizontale Textzeile oben:

“Selbst Entstandener, Vorfahr, der dies alles machte, Heilige Schlange, auf der Sia ist, ewig Lebender, Unverganglicher, der, aus dessen Darm Wind und aus dessen Nase der Nordwind kommt.”

Titulatur des Kônigs:

Beischrift zur Sonnenscheibe:

"Der von Edfu"

Südturturm, Ostseite

Drei Register sind erhalten, davon ist das oberste fast ganz fortgebrochen. Darstellungen wie auf dem Nordturm.

Oberstes Register:

Der Kônig steht vor einem Gott. Der Rest der Darsellung ist zestört.

Text vor dem Kônig:

(...) ( damit du) ihren Duft reichst (...) fûr deinen ka als Wohlgeruch (jd.t)

Deiner Glieder, dieser Duft, der stammt."

Text vor dem Gott:

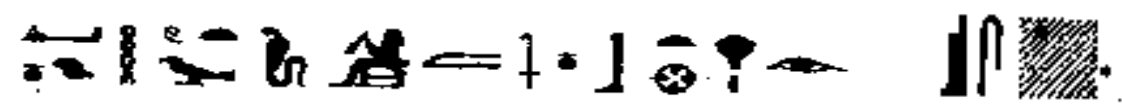

"Ich gebe dir prächtige Heweret ${ }^{10}$ um ihren Platz an deinem Ort einzurichten."

1. Register:

Der Kõnig mit Atefkrone, erhebt ein Sistrum vor x und y.

Horizontale Textzeile oben:

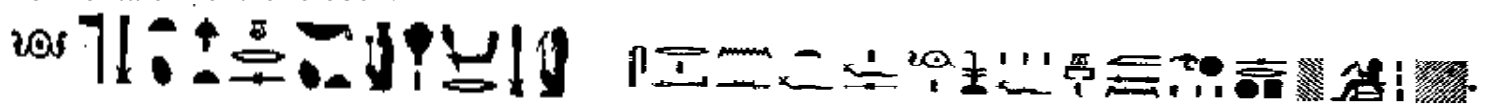

“ Die Gottesschwester, die Treffliche, Leiterin der Frauen, die ihren Bruder

schutz, der ihr Vater Re

Horizontale Textzeile oben:

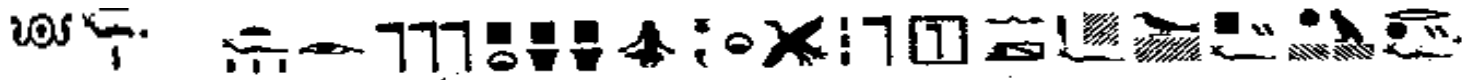

“Vater der Väter, der die Neunheit erschuf und die Uranfanglichen gaber, heiliger Gott, jener Grosse, dessen Gestalt unsicher ist und dessen Be schaffenheit (rx) unbekannt ist."

Titulatur des Kõnigs:

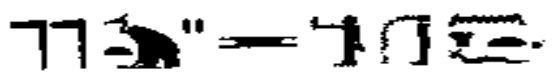

" Theoi Philometores, der das Opfer fur seinen Herrn vermehrt."

Titulatur des Amun:

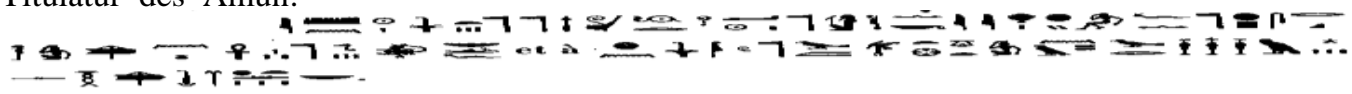

“ Amun- Re, Kônig der Gõtter, grosser Gott von Urbeginn an, einziger Gott, der als Jungling nicht seinesgleichen hat. Es gibt keinen Gott, der ihm Gleicht, Lebenshauch der Gõtter, durch den jedermann atmen."

Titulatur des Chons:

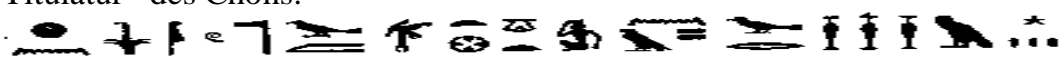

"Chons- Schu, Grosser in Theben, der sich auf seinem Platz als Re- Harachte befindet, gross an Beliebheit unter den Gõttern, der den Lebenshauch dauern lasst, bleibend an allen guten Dingen.”

Text vor dem Kõnig:

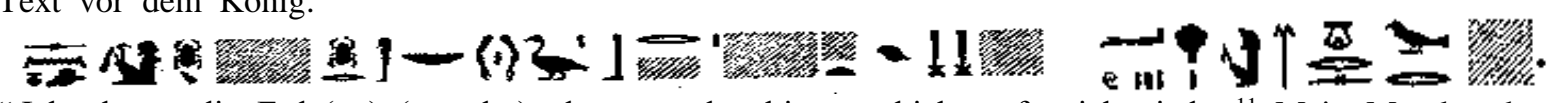

"Ich kusse die $\operatorname{Erde}(\ldots)$ (aus der) du entstanden bist, und ich werfe mich nieder. ${ }^{11}$ Mein Mund steht unter deiner Wahrheit (sfj.t), und ich beuge meine Arme."

Text vor Amun:

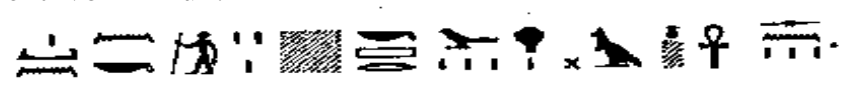

"Ich gebe dir die Grossen (...) und die Geringen geben dir Lobpreis,, auf das sie leben warden."

Text vor Chons:

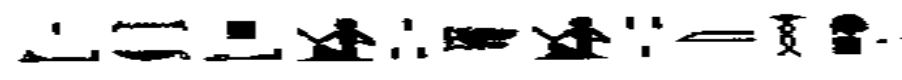

“ Ich gebe dir die $\mathrm{P}<. \mathrm{t}$ und die Rxj.t mit demutigeneigtem Haupt ( $\mathrm{m}$ w3h tp)." 
Zweite Tor

\section{INSCHRIFTEN DES ZWEITEN UND DRIITEN \\ TORE DES PTAHTEMPEL IN KARNAK \\ von \\ MAHMUD ABDEL RAZIQ}

Südturm Ostseite

Das zweite Tor, aus der Zeit der 25./26. Dynastie, ist deutlich kleiner als das erste. Die Westseite ist auf beiden Turmen mit mehreren kleinformatigen Registern dekoriert, die fast durchgehend lediglich das Bild eines sitzenden Gottes tragen. Die Tecknik ist kräftiges Flachrelief. Die seitliche Registerbegrenzung erfolgt jeweils vorne durch eine von oben nach unten durchgehende jahresrispe, die auf dem Kopf eines heh-Gottes ruht; und jeweills hinten durch ein von oben nach unten durchgehendes w3s- Szepter. Dazu verläuft an der Durchgangsseite auf beiden Turmen eine senkrechte Textzeile."

Zweites Tor

Nordturm, Westseite:

Oberstes Register:

Text: :

"(...) wie Re"

2.Register:

Text:

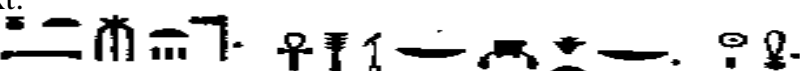

"Nut, die die Gõtter. Sie mogen alle Gesundheit, Freude und Dauer, alles Leben und Wohlergehen geben wie Re"

3.Register:

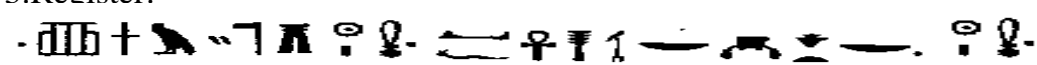

"Chontamenti, er möge alle Gesundheit, alles Leben und Wohlergehen, alle Freude gegeben wie Re." 4.Register:

Text:

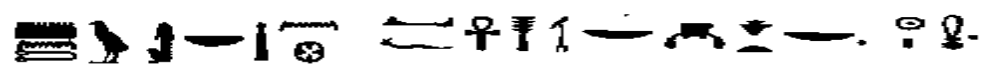

"Month, Herr von Hermonthis, er mõge alles Leben und Wohlergehen alle Freude geben wie Re." 5.Register:

Text

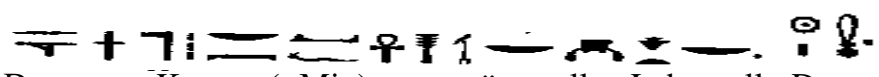

“ Der- von Koptos (=Min), er möge alles Leben alle Dauer, alles Wohlergehen und alle Freude, alle Freude geben wie Re."

6.Register:

Text:

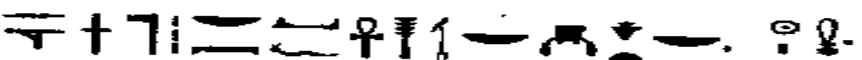

"Min, der sich unter den Gõttern befindet, Herr des Himmels, er mõge alles Leben, alle Dauer, alles Wohlergehen und alle Freude geben wie Re."

Unter Register:

Text:

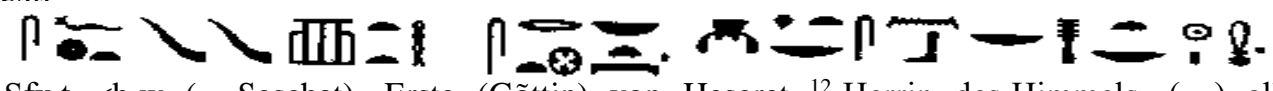

"Sfx.t- <b.w ( = Seschat), Erste (Gõttin) von Heseret, ${ }^{12}$ Herrin des Himmels, (...) alle Freude, Gesundheit und Dauer geben wie Re." Dahinter steht ein Mann mit einem rechteckigen Gegenstand in der Hand:

$$
17 \text { 우은 }
$$
Text:

“ Der lebende Kõnigs-ka, Herr aller Freude, Gesundheit und Dauer wie Re.

"Senkrechte Zeile:

“(...) alle Annalen als Hebsed- Feste, Indem du erscheinst als Kõnig von Ober- und Unterägypten auf dem Thron des Horus und du alle Menschen ( leitest ) wie Re, ewiglich."

Oberstes Register:

Text:

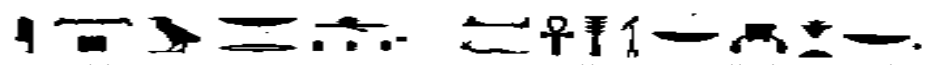

"Anubis, Herr von Rosetau, er möge alle Gesundheit, Freude und Starke geben wie Re." 2.Register:

Text:

\section{$01 \div 2$ 우}

"Sobek, Herr des Fajums (?0, ist der grosse Gott, er mõge alles Leben und Wohler- geben (...)."

Zweites Tor

Sûdturm, Westseite:

3. Reoister

\section{恶}

"Wennenefer- Chontamenti (=Osiris), der grosse Gott, (....) wie Re."

4.Regiter: 
Text:

"Chnum von Elephantine ( $3 \mathrm{bw}$ ), grosser Gott, er môge alle Freude geben wie Re."

Unterstes Register:

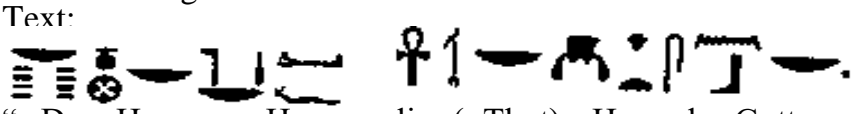

"Der Herr von Hermopolis (=Thot), Herr der Gottesworte, er môge alles Leben und Wohlergehen, alle Freude und Gesundheit geben."

Beischrift 7.11 der Figiir hinter dem Gott:

\section{$121+701$ 우}

“Der lebendige Kônigs-Ka, Herr der beiden Länder, der seinem Herrn Leben gibt."

Senkrechte Zeile:

Mit Hebsed Festen, indem du erscheinst auf dem Thron des Horus als Kõnig von Ober- und Unterägypten (und

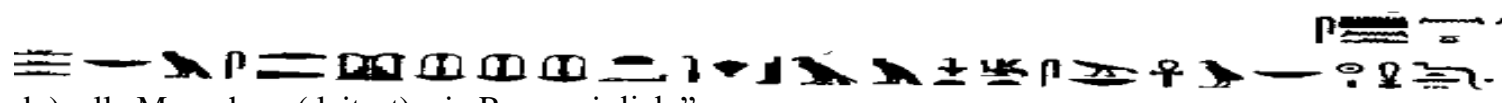

du) alle Menschen ( leitest) wie Re, ewiglich."

Zweites Tor

Nordturm, innen, Westlich der Nische:

Eine senkrechte Textzeile mit der Titulatur des Schabaka:

Südturm, Innen, Westseitlich der Nische:

Eine senkrechte Textzeile mit Titulatur des Schabaka:

Nordturm, Nische:

Die Nische enthalt zwei Register, in denen der Kõnig jeweils vor mehreren Gõttern opfert

Oberes Register:

Schabaka erhebt zwei Weingefässe vor Amun, Mut, Ptah, Hathor und Amaunet.

Titulatur des Kõnigs:

士

\section{$[<1=[\equiv$}

Horus ,sqb-t3wy, Kõnig von Ober- und Unterägypten Nfr-k3-R<, Sohn des Re (Schabaka), geliebt von Hathor, begabt mit Leben, ewiglich."

Uber dem Kõnig: " der grosse Gott, er mõge leben wie Re"

Text vor dem Kõnig:"Seinem Vater Wein darreichen"

Titulatur des Amun:

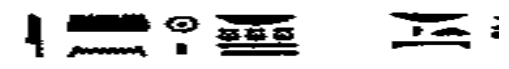

"Amun- Re, Herr von Karnak, Herr des Himmels"

Text vor Amun:

우 $1-1$ i

"Rede: Ich gebe dir alles Leben und Wohlergehen" "Rede: Ich gebe dir alle Gesundheit"

$-2+2$

Titulatur des Ptah:

"Mut, Herrin des Himmels"

"Ptah, Herr der Maat, Herr allen Leben"

Text vor Ptah:

드 $=-8=\frac{1}{=}$.

“ Rede·Ptah. Herr der Maat Herr allen Leben."

\& $1-n=1-$

"Rede: Ich gebe dir alles Leben und alle Gesundheit, und alle Freude."

Zweites Tor

Nordturm, Nische:

Titulatur der Hathor:-

$-\square=\frac{5}{6}=$

"Hathor, in Theben befindlich..."

Text Vor Hathor:

$$
\text { 우 } 1-1 \text { I }
$$

"Rede : Ich gebe dir alle Gesundheit, alle Freude."

Titulatur der Amaunet:

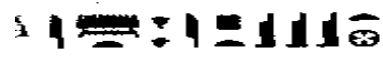

"Amaunet, in Karnak befindlisch..."

Text vor Amaunet:

\& $1=0 \frac{1}{1}-1$

"Rede: Ich gebe alles Leben und Wohlergehen, alle Gesunheit und jegliche Starke (...)."

Unteres Register:

Die Darstellungen diese register sind stark zerstõrt; Sie zeigen den Kõnig, links, vor einer Gruppe von Gõttern. Erkennbar sind Re-Harachte, Amun (?) und Ptah. Sie blacken nicht alle zum Kõnig, wie oben, sondern gruppieren sich um die Gestalt sitzenden Amun (?) in der Mitte. 
Südturm, Nische:

Keine Angaben. Zerstõrt?

Nordturm, Ostseite (2.seite)

Drittes Tor

Südturm, Ostseite:

Dies ist ein schmales Gewände mit den Massenx.x.x m

Erhaltene Hohe links:

Erhaltene Hohe rechts:

Baudatum Ptol. X11l.

Die Westseiten und die westlichen Gewände der Innenseiten sind mit senkrechten Inschriften.

Nordturm, Westseite:

Nordturm, Westseite Zwei einanderüberliegende Inschriftenzeilen.

I inks

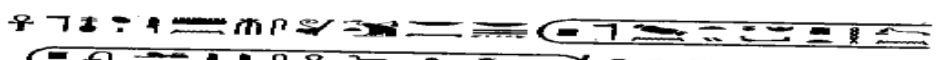

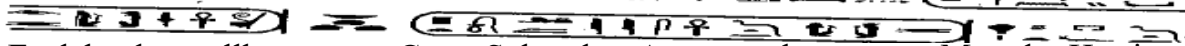

“Es lebe der vollkommene Gott, Sohn des Amun, geboren von Mut, der Herrin des Himmels, der Herr der beiden Länder, Naos Dionysos, Herr der Kronen, Ptolemaios (X11l) auf seinem Thron, ewiglich." Rechts:

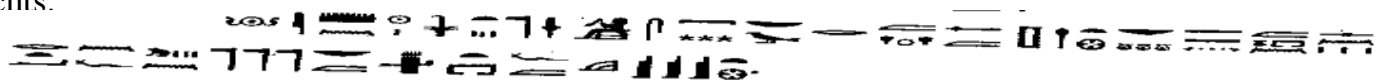

“Amun- Re, Kõnig der Gõtter, prächtiges Abbild, der uber allen Gõtter steht, Grosser in Heliopolis, Erhabener in Memphis, Herr von Karnak in ihrem Inneren, den Tausende von allen Gõttern in Palast im Tempel von Karnak gesetzt haben".

Südturm, Westseite:

Südturm, Westseite Zwei einander gegenüberstehende Inschriftzeilen.

I inks:

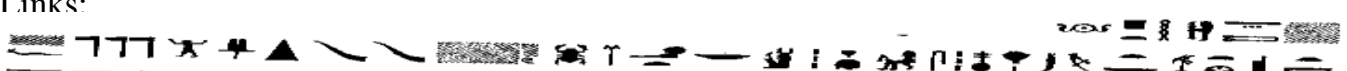

$==$

“Ptah- Tatenen (...) die Gõtter, mit hohem Federnpaar und spitzen Hornern (...) ist er, der am Urbeginn entstand, Herr der Heka(krafte), Oberster der Vornehmen, Schõngesichtiger, Liebreizender, Herr von Theben, dem Ort den er liebt."

Rechts:

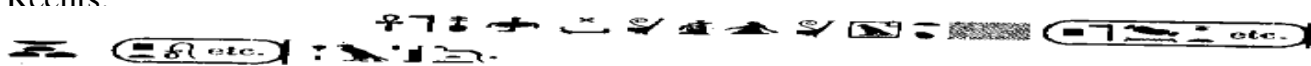

"Es lebe der vollkommene Gott, der von Ptah erschaffen und von Hathor aufgezogen wurde (...) Neos

Dionysos,, Herr der Kronen, Ptolemaios (X1ll), auf dem Thron des Horus, ewiglich."

Bildfeld oberhalb der Zeilen:

Ptolemaus Xlll Neos Dionysos als Sphinx mit Dopelkrone reicht dem Ptah ein Gefäss dar.

Titulatur des Ptah:

“Ptah Herr $\bar{x} 7 \mathbf{1}=$

"Ptah, Herr von Theben, grosser Gott, Herr der Maat."

Nordturm, innen, westliches Gewand:

Fine senkrechte Inschriftzeiler mit der Titulatur der Mut

"Mut, Herrin von Ascheru, Mutter der Mutter, die jeden Gott gebar, grosse Sternschlange, die ihren Vater Re umgibt, den sie als Chons auf die Erde gegeben hat. Sie gewährt die Lebenszeit des Re im Himmel dem Herrn der beiden Länder Neos Dionysos."

Südturm, innen, westliches Gewände:

Fine senkrechte Inschriftzeile mit Titulatur der Hathor

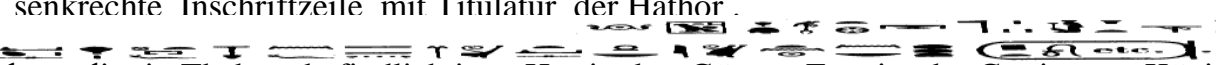

"Hathor, die in Theben befindlich ist, Herrin der Gõtter, Fürstin der Gottinnen, Kõnigin der Untertanen, die ihren Sohn auf seinen Thron gesetzt hat, damit er die beiden Länder durch die Doppelkrone vereine. Sie gibt all das, was die Sonne umkreist ( sn.w nb nj jtn) dem Herrn der Kronen Ptolemaios (X11l)."

Vor dem Tor auf dem Boden liegend: Ein Architravfragment. Aufschrift ? Zugehõrigkeit ?

$$
\begin{aligned}
& \text { معبد بتاح في الكرنك } \\
& \text { يتعلق البحث بمجموعة مقالات عن معبد بتاح والمقالتين الأخيريتين عن الصالة التى تسبق قدس الأقداس فى المعبد. }
\end{aligned}
$$

\footnotetext{
2 Name eines fremden Volkes in Nubien ( WB 1, 116.1)

${ }^{3}$ S. WB 111, 402.9

${ }^{4}$ Ergänze <ntjw nach MARIETTE, Denderah 1 pl. 31,8

${ }^{5}$ So nach MARIETTE, Denderah $1 \mathrm{pl}, 31,8$

${ }^{6}$ Wortl.: "Sein Leib ist offen"

${ }^{7}$ Ergänze nach MARIETTE, Denderah 1, pl. 31 11/11

${ }^{8}$ d.h. gegen das Unheil.

${ }^{9}$ hmt. r3, s. WB 11185.2

10 (hwr.t), Beiname der Geiergõttin von Elkab.

${ }^{11}$ Wortl. : “ Geb umfassen”, WB 111, 72.5

${ }^{12}$ Hsr.t Name der Nekropole von Hermopolis
}

${ }^{1}$ HIER UND FOLENDEN DAS BILD DER SONNENSCHEIBE EINFUGEN 